\title{
Évolution de la régénération après la coupe de peuplements récoltés selon différents procédés d'exploitation
}

\author{
par David Pothier
}

Entre 1982 et 1984, 289 blocs échantillons ont été établis dans autant d'aires de coupe distribuées dans la toute la zone des forêts mixtes et résineuses du Québec. Les inventaires de régénération réalisés avant et après la coupe ont permis de déterminer le pourcentage de la régénération détruite par quatre procédés d'exploitation différents. De plus, l'évolution de la régénération à moyen terme a pu être documentée à partir d'inventaires effectués cinq et dix ans après la coupe. Pour l'ensemble des peuplements échantillonnés, les procédés d'exploitation ont abaissé le coefficient de distribution de la régénération (CDR) de 25 à $40 \%$. La plus faible baisse de CDR est associée à l'utilisation d'une abatteuseporteuse qui semble plus efficace pour protéger les semis d'une hauteur inférieure à $50 \mathrm{~cm}$ comparativement aux procédés utilisant des débusqueuses à câble. L'effet de cette meilleure protection de la régénération n'est cependant plus apparent cinq ans après la coupe à cause du recrutement de nouveaux semis observé dans la plupart des peuplements. Par rapport à la composition du peuplement original, le sapin a tendance à augmenter sa proportion dans la strate de régénération parce qu'il s'établit facilement sous le couvert du peuplement mûr. La composante d'espèces feuillues a aussi tendance à augmenter par rapport au peuplement original de sorte que certains peuplements résineux évolueraient vers des peuplements mélangés si la composition des peuplements à maturité était fidèle à la composition des tiges dominantes retrouvées 10 ans après la coupe. La remise en production des peuplements de pin gris semble problématique à cause de la rareté de la régénération préétablie qui est, de surcroît, partiellement détruite lors de l'exploitation. La plantation, le regarni ou l'ensemensement pourrait donc être envisagés dans ces types de peuplement mais la coupe avec protection de la régénération devrait être appliquée dans les autres peuplements pour préserver la régénération préétablie et particulièrement les individus de haute taille.

Mots clés: coupe à blanc, régénération préétablie, procédé de récolte, débusqueuse à câble, abatteuse-porteuse
Between 1982 and 1984, 289 sample plots were established on cutovers distributed across the mixed and the boreal forest zones of Québec. Regeneration surveys before and after clearcutting were used to determine the proportion of regeneration destroyed by four different harvesting systems. Moreover, information on the evolution of the regeneration over the medium term was gathered from surveys realized five and ten years following logging. For all stands combined, harvesting resulted in a reduction of 25 to $40 \%$ of the stocking of the regeneration. The lowest decrease in stocking was produced by a feller-forwarder that was more efficient to protect regeneration shorter than $50 \mathrm{~cm}$ as compared to harvesting systems using cable skidders. Differences between harvesting systems were no longer apparent after the first fiveyear-period following logging because establishment of new seedlings was observed in most of the sampled cutovers. The ability of balsam fir to establish under a canopy generally caused an increase of its proportion in the new stands as compared to the original stands. The proportion of hardwood species also tended to increase in comparison to the original stand in such a way that some conifer stands would evolve toward mixed stands if no change in stand composition occurred after the first ten-year period following logging. Natural regeneration of jack pine stands after harvesting seems difficult to obtain since these stands have scarce advance regeneration that is partially destroyed during logging operations. Plantation, fill plantation or seeding could be applied in these stands but harvesting methods providing protection for regeneration should be used in the other stands in order to protect advance regeneration and particularly tall individuals.

Key words: clearcutting, advance regeneration, harvesting system, cable skidders, feller-forwarder

\section{Introduction}

La mécanisation des opérations forestières qui s'est réalisée au cours des dernières décennies a soulevé plusieurs questions concernant la destruction de la régénération préétablie et le recrutement de semis s'établissant après la coupe. Ce questionnement semble justifié dans le cas des coupes à blanc exécutées sur de grandes superficies à cause de la rareté des arbres semenciers laissés sur le parterre de coupe. Les études entreprises sur le sujet ont révélé que les coupes mécanisées conventionnelles pouvaient réduire le coefficient de distribution de la régénération $(\mathrm{CDR})$ préétablie dans une proportion variant de 10 à $60 \%$ en fonction, notamment, du type de peuplement et de station, de la densité de la régénération prétablie,

Direction de la recherche forestière, Ministère des Ressources naturelles du Québec, 2700, rue Einstein, Sainte-Foy, Québec, Canada G1P 3W8. du type de machinerie utilisée, des habitudes de travail des opérateurs de machinerie et de la saison de récolte (Arnott 1968; Webber et al. 1969; Frisque et al. 1978; Froning 1980; Gingras 1990; Gingras et al. 1991; Ruel 1992; Pominville 1993; Groot 1995; McInnis et Roberts 1994). Il semble que la destruction de la régénération se fasse principalement dans les sentiers de débardage mais aussi à leurs abords lorqu'ils sont utilisés de façon répétée (Webber et al. 1969; Gingras 1990; Gingras et al. 1991). De plus, ces auteurs mentionnent que le balayage des arbres attachés aux débusqueuses à câble peut aussi occasionner une certaine mortalité de la régénération. La destruction de la régénération préétablie ne semble pas être compensée par la formation de lits de germination propices à l'établissement de nouveaux semis puisque les superficies ainsi perturbées ne représentent qu'un très faible pourcentage des aires de coupe (Arnott 1968; Webber et al. 1969; Frisque et al. 1978; Martin 1988). 
Il ne faudrait toutefois pas conclure hâtivement de ces études que la mécanisation des opérations forestières cause un tort irrémédiable à la régénération désirée et, par conséquent, à la production des futurs peuplements. En effet, il est possible de remettre adéquatement en production les aires de coupe en utilisant des appareils adaptés aux conditions particulières des stations pour protéger la régénération (Gingras 1990; Gingras et al. 1991). Par exemple, il serait préférable d'utiliser des porteurs forestiers plutôt que des débusqueuses à câble sur les sols possédant une bonne capacité portante (Frisque et al. 1978; Gingras 1990; Gingras et al. 1991). De plus, des méthodes de travail ont été mises au point pour réduire les risques de dommage à la régénération tout en n'affectant que légèrement la productivité des opérations (Gingras 1990; Gingras et al. 1991). C'est le cas notamment de l'établissement d'un patron de circulation sur le parterre de coupe, de l'espacement maximal entre les sentiers de débardage et de l'abattage ou de l'empilement des arbres selon un angle se rapprochant de l'axe des sentiers de débardage. Ces mesures de protection de la régénération ont été mises au point parce qu'une proportion importante des arbres d'avenir identifiés après la coupe est issue de la régénération préétablie (Webber et al. 1969; Frisque et al. 1978; Ruel 1989; Ruel 1992; Pominville 1993; Ruel et Huot 1993). La plus haute taille initiale de la régénération préétablie comparativement aux semis établis après la coupe pourrait lui permettre de se libérer plus rapidement des espèces compétitrices (Hatcher 1960 ) et éventuellement devancer l'âge de récolte des futurs peuplements (Pothier et al. 1995).

Le développement de la régénération et l'évolution de la composition des peuplements après la coupe constituent aussi des sujets d'intérêt pour les aménagistes forestiers. En effet, dans le contexte d'un aménagement visant le rendement soutenu, il devient important de prélever périodiquement le même volume des différentes espèces en présence afin de subvenir aux besoins spécifiques des industries dépendantes d'un territoire d'approvisionnement. Une façon simple d'atteindre cet objectif consisterait à ce que la distribution et la composition de la strate de régénération soient semblables à celles du peuplement récolté. Toutefois, certaines études font état d'une mauvaise distribution de la régénération et d'une augmentation substantielle de la proportion d'espèces feuillues dans la strate succédant à la coupe de peuplements résineux (Frisque et al. 1978; Ruel 1992; Pominville 1993; Ruel et Huot 1993). D'autres auteurs ont observé que la proportion de sapin baumier (Abies balsamea [L.] Mill.) avait tendance à augmenter au détriment des épinettes lorsqu'on comparait la composition du peuplement récolté à celle de la strate de régénération (Hatcher 1960; Fye et Thomas 1963; Vézina et Falardeau 1988). Ainsi, pour certains types de peuplement, la similitude des strates récoltées et régénérées en termes de production et de composition semble constituer un objectif d'aménagement parfois difficile à atteindre.

Dans le but d'apporter des éléments de réponse aux questions soulevées par les praticiens forestiers, une étude a été entreprise en 1982 dans des aires de coupe représentatives des procédés d'exploitation utilisés au Québec à cette époque. L'objectif premier de cette étude était de documenter l'effet des procédés de récolte alors utilisés sur la destruction de la régénération préétablie et sur le développement des peuplements après la coupe. La prise de données avant la coupe a permis de faire le lien entre les caractéristiques des stations et la composition et l'abondance de la régénération préétablie (Ruel 1989). Les premiers inventaires réalisés après la coupe ont fait ressortir l'importance de la régénération préétablie pour succéder à la récolte de peuplements d'épinette noire (Picea mariana [Mill.] B.S.P.) et de sapin baumier (Ruel 1992; Ruel et Huot 1993). Le présent ouvrage traite de l'évolution décennale de la composition des peuplements nouvellement formés en fonction des procédés de récolte et des groupements d'espèces initiaux.

\section{Matériel et méthodes}

De 1982 à 1984, plus de 400 aires de coupe à blanc ont été sélectionnées dans toute la zone des forêts mixtes et résineuses du Québec pour être mesurées périodiquement au cours des 10 premières années suivant la coupe. Pour diverses raisons, plusieurs blocs d'étude ont dû être abandonnés au fil des ans de sorte que le présent rapport traite des données obtenues de 289 des 424 blocs initialement établis.

Chaque bloc d'étude, d'une superficie de deux hectares, a été délimité avant la coupe pour caractériser le peuplement récolté. Ainsi, des renseignements sur la topographie, le drainage et le sol ont été recueillis pour définir l'environnement physique des aires d'étude. Deux parcelles échantillons circulaires d'une superficie de $400 \mathrm{~m}^{2}$ ont aussi été établies avant la coupe dans chaque bloc d'étude pour y dénombrer les arbres marchands (DHP > $9,1 \mathrm{~cm}$ ) par classe de diamètre de deux $\mathrm{cm}$ et par espèce. Cette prise de données a permis de calculer la surface terrière de chaque espèce et de déduire le groupement d'espèces caractérisant les peuplements (tableau 1) selon les critères de stratification présentement en vigueur au Québec (Ministère des Ressources naturelles du Québec 1995).

La prise de données avant la coupe était complétée en établissant 98 placettes de $4 \mathrm{~m}^{2}$ disposées systématiquement de façon à couvrir l'ensemble du bloc d'étude. Dans chaque placette de $4 \mathrm{~m}^{2}$, des renseignements ont été pris concernant la présence ou l'absence de semis de sapin baumier, d'épinette blanche (Picea glauca [Moench] Voss), d'épinette noire, de pin gris (Pinus banksiana Lamb.), de bouleau jaune (Betula alleghaniensis Britt.), de bouleau à papier (Betula papyrifera Marsh.), de peuplier fauxtremble (Populus tremuloides Michx.) et de l'ensemble des autres espèces arborescentes. Une classe de hauteur était assignée aux semis en ne considérant que la plus haute tige de chaque espèce par placette. Pour chaque bloc d'étude, il a donc été possible de calculer le coefficient de distribution de la régénération (CDR) par classe de hauteur pour l'ensemble des espèces et pour chacune d'elles prise individuellement. De plus, un dénombrement des semis par espèce a été effectué dans 33 des 98 placettes de $4 \mathrm{~m}^{2}$ des blocs d'étude.

Après que les peuplements aient été récoltés selon les procédés d'exploitation décrits au tableau 2, la prise de mesure des placettes de régénération s'est répétée à trois reprises, soit un an, cinq ans et 10 ans après la coupe. Les prises de mesure effectuées avant la coupe et un an après ont permis de calculer la diminution relative du coefficient de distribution de la régénération de la façon suivante:

$$
\frac{\text { CDR avant coupe }- \text { CDR après coupe }}{\text { CDR avant coupe }} \times 100
$$

Lors de la quatrième prise de mesure (10 ans après la coupe), des informations supplémentaires ont été recueillies dans chaque placette de régénération. Ainsi, la hauteur et la crois- 


\begin{tabular}{|c|c|c|c|c|c|c|c|}
\hline $\begin{array}{l}\text { Groupement } \\
\text { d'espèces }\end{array}$ & $\begin{array}{l}\text { Nombre } \\
\text { d'aires } \\
\text { d'étude }\end{array}$ & $\begin{array}{c}\text { Surface } \\
\text { terrière } \\
\text { totale } \\
\left(\mathrm{m}^{2}\right)\end{array}$ & $\begin{array}{c}\text { Surface } \\
\text { terrière } \\
\text { Pig } \\
\left(\mathrm{m}^{2}\right) \\
\end{array}$ & $\begin{array}{c}\text { Surface } \\
\text { terrière } \\
\text { Epn } \\
\left(\mathrm{m}^{2}\right) \\
\end{array}$ & $\begin{array}{c}\text { Surface } \\
\text { terrière } \\
\text { Sab } \\
\left(\mathrm{m}^{2}\right) \\
\end{array}$ & $\begin{array}{c}\text { Surface } \\
\text { terrière } \\
\text { Bop } \\
\left(\mathrm{m}^{2}\right) \\
\end{array}$ & $\begin{array}{c}\text { Surface } \\
\text { terrière } \\
\text { Pet } \\
\left(\mathrm{m}^{2}\right) \\
\end{array}$ \\
\hline $\mathrm{PgPg}$ & 9 & $16,3 \pm 1,1$ & $14,9 \pm 0,8$ & $1,3 \pm 0,3$ & - & $0,1 \pm 0,1$ & $0,1 \pm 0,1$ \\
\hline $\mathrm{PgE}$ & 13 & $22,7 \pm 1,8$ & $13,7 \pm 1,3$ & $6,1 \pm 0,8$ & $0,6 \pm 0,4$ & $1,7 \pm 0,6$ & $0,3 \pm 0,1$ \\
\hline $\mathrm{EPg}$ & 8 & $19,7 \pm 2,4$ & $6,5 \pm 1,1$ & $12,6 \pm 1,6$ & $0,1 \pm 0,1$ & $0,6 \pm 0,4$ & $0,1 \pm 0,1$ \\
\hline $\mathrm{EE}$ & 112 & $21,7 \pm 0,7$ & $0,4 \pm 0,1$ & $19,9 \pm 0,7$ & $1,0 \pm 0,1$ & $0,4 \pm 0,1$ & $0,1 \pm 0,1$ \\
\hline ES & 22 & $23,0 \pm 1,6$ & $0,2 \pm 0,1$ & $14,0 \pm 1,1$ & $6,7 \pm 0,8$ & $1,0 \pm 0,3$ & $0,1 \pm 0,1$ \\
\hline SE & 28 & $24,5 \pm 1,3$ & - & $8,0 \pm 0,5$ & $14,4 \pm 1,1$ & $1,1 \pm 0,3$ & $0,1 \pm 0,1$ \\
\hline SS & 76 & $32,4 \pm 1,0$ & - & $1,6 \pm 0,3$ & $25,6 \pm 1,0$ & $1,6 \pm 0,2$ & $0,1 \pm 0,1$ \\
\hline $\mathrm{BbE}$ & 4 & $19,7 \pm 3,5$ & $1,6 \pm 1,0$ & $5,4 \pm 1,5$ & $0,4 \pm 0,3$ & $10,9 \pm 2,4$ & $0,2 \pm 0,2$ \\
\hline $\mathrm{EBb}$ & 5 & $16,2 \pm 3,3$ & - & $8,7 \pm 3,2$ & $1,7 \pm 0,5$ & $5,1 \pm 0,8$ & - \\
\hline $\mathrm{BbS}$ & 2 & $23,0 \pm 1,3$ & - & $2,4 \pm 2,4$ & $6,2 \pm 0,1$ & $12,8 \pm 0,7$ & - \\
\hline $\mathrm{SBb}$ & 5 & $24,4 \pm 3,2$ & $0,4 \pm 0,2$ & $3,0 \pm 1,2$ & $10,7 \pm 3,0$ & $8,1 \pm 1,0$ & $0,3 \pm 0,2$ \\
\hline $\mathrm{PgPe}$ & 2 & $25,4 \pm 2,0$ & $10,3 \pm 1,8$ & $4,8 \pm 2,0$ & - & $1,2 \pm 1,2$ & $8,8 \pm 3,8$ \\
\hline PeS & 3 & $22,0 \pm 9.6$ & $1,3 \pm 1,3$ & $1,1 \pm 0,4$ & $4,0 \pm 2,0$ & $3,8 \pm 1,7$ & $9,8 \pm 4,3$ \\
\hline
\end{tabular}

Note: Pig, Pg, pin gris; Epn, E, épinette noire; Sab, S, sapin baumier; Bop, Bb, bouleau à papier; Pet, Pe, peuplier faux-tremble. La codification des groupements d'espèces est conforme aux normes de stratification écoforestière utilisées au Québec (Ministère des Ressources naturelles du Québec 1995).

\begin{tabular}{l}
\hline Tableau 2. Liste des procédés de récolte utilisés \\
\hline Procédé 1: Abatteuse-porteuse (arbre entier) \\
Procédé 2: Abatteuse mécanique, débusqueuse à câble (arbre entier) \\
Procédé 3: Abattage manuel, débusqueuse à câble (arbre entier) \\
Procédé 4: Abattage et ébranchage manuel, débusqueuse à câble (bois en \\
$\quad$ longueur)
\end{tabular}

sance en hauteur des plus hautes tiges commerciales résineuses et feuillues de même que la hauteur et le pourcentage de couverture des espèces non commerciales ont été notés. Ces mesures de hauteur ont permis de déterminer l'espèce dominante de chaque placette de régénération, ce qui ne pouvait pas toujours être fait lors des inventaires précédents puisque seules les classes de hauteur étaient alors notées.

Pour chacune des quatre prises de mesure, les données ont été soumises à des analyses de variance selon un dispositif complètement aléatoire pour détecter des différences entre les procédés de récolte. Lorsque les analyses de variance indiquaient une différence entre les moyennes obtenues des différents procédés $(\mathrm{p}<0,05)$, le test de comparaisons multiples de Tukey a été utilisé.

\section{Résultats et discussion}

\section{Effets des procédés d'exploitation}

Pour l'ensemble des peuplements échantillonnés, les procédés d'exploitation ont abaissé le coefficient de distribution de la régénération de 25 à $40 \%$ (figure 1), ce qui correspond aux baisses de CDR observées dans d'autres études portant sur le même type de système de récolte (Arnott 1968; Webber et al. 1969; Froning 1980; Gingras et al. 1991; Pominville 1993; Groot 1995). Cet ordre de grandeur de destruction de la régénération est caractéristique d'un mode de débardage sans patron de circulation défini. Il est cependant possible de réduire les pertes de régénération en diminuant les superficies couvertes par les sentiers de débardage (Webber et al. 1969; Froning 1980; Gingras et al. 1991; Pominville 1993).

La plus faible baisse du CDR est associée à l'utilisation d'une abatteuse-porteuse (procédé 1) quoique ce procédé ne se distingue pas significativement des autres $(\alpha<0,05)$ lorsque toutes les classes de hauteur de la régénération sont regroupées (figure 1). Toutefois, lorsque seuls sont considérés les semis de 5 à $50 \mathrm{~cm}$ de hauteur, la baisse du CDR associée au procédé utilisant l'abatteuse-porteuse est significativement inférieure à la baisse liée aux autres procédés qui ont tous utilisé des débusqueuses à câble (figure 1). Cette meilleure protection de la régénération par un porteur forestier comparativement à une débusqueuse à câble a déjà été signalée par Frisque et Vézina (1977), Gingras (1990), et Ruel (1992). Les débusqueuses à câble sont sujets à causer plus de dommages à la régénération que les porteurs parce qu'ils provoquent un balayage du sol par les arbres qui ne sont pas disposés parallèlement à l'axe des sentiers. De plus, dans le cas d'une abatteuse-porteuse (procédé 1), des avantages supplémentaires découleraient du fait que les arbres ne touchent pas au sol entre l'abattage et le chargement et que les déplacements de la machinerie sont diminués puisqu'un seul appareil est nécessaire pour abattre et emporter les arbres hors de l'aire de coupe (Gingras 1990). Ce dernier auteur précise toutefois que les fortes dimensions des abatteusesporteuses pourraient atténuer le précédent avantage en accentuant les dommages causés à la régénération située en bordure des sentiers surtout si la récolte du bois se fait sur des stations humides ou sur des pentes fortes.

Pour les semis de hauteur supérieure à $50 \mathrm{~cm}$, la diminution relative du CDR n'a pas été significativement modifiée $(\alpha=0,05)$ par les procédés de récolte (figure 1). Cependant, la destruction de la régénération s'est intensifiée parallèlement à l'augmentation de la classe de hauteur de la régénération peu importe le procédé de récolte utilisé (figure 1). Puisque tous les procédés de récolte ont utilisé de la machinerie lors de l'abattage ou du débardage (tableau 2), la destruction de la régénération de haute taille semble donc imputable aux déplacements de ces véhicules sur le parterre de coupe. Il est probable que le diamètre des roues des abatteuses et des débardeurs permette à la régénération de petite taille de se glisser sous les véhicules sans subir de dommages importants alors que les tiges plus hautes seraient fortement abîmées ou renversées. Il serait pourtant important d'établir des mesures de protection de ces plus hauts individus de la régénération préétablie car ils peuvent constituer les sujets dominants du futur peuplement et contribuer à en augmenter la production ligneuse à maturité (Pothier et al. 1995). 


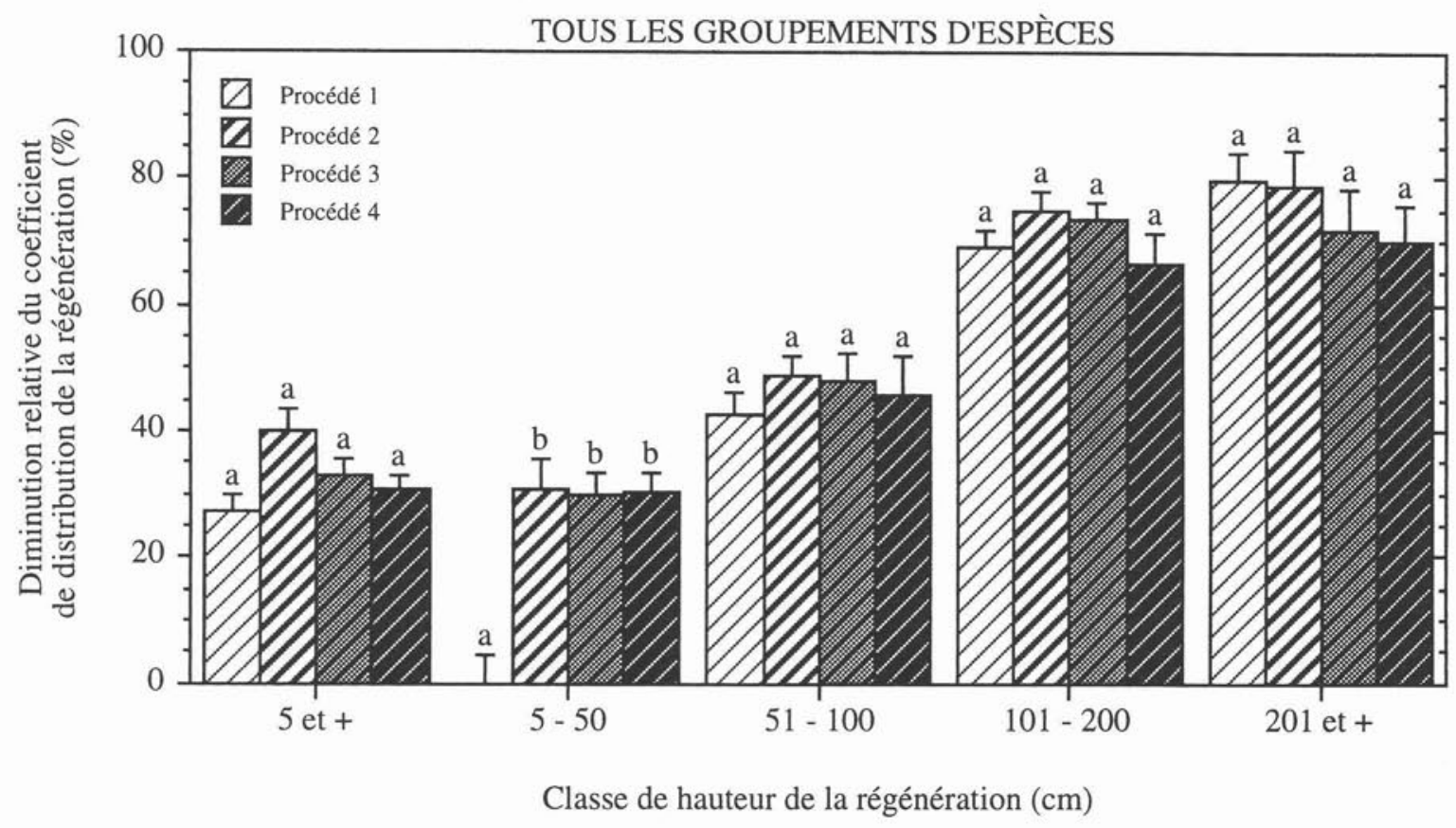

Fig. 1. Diminution relative du coefficient de distribution de la régénération par classe de hauteur de la régénération pour les quatre procédés de récolte étudiés dans l'ensemble des blocs expérimentaux. Pour chaque classe de hauteur, des lettres différentes indiquent que les moyennes associées aux procédés de récolte sont significativement différentes selon le test de comparaison multiple de Tukey $(\alpha=0,05)$.

\section{2. Évolution de la régénération dans les pessières à épinette noire pures}

De façon à isoler la persistance de l'effet du procédé de récolte sur l'évolution de la distribution et de la composition de la régénération, les pessières à épinette noire pures (groupement d'espèces EE) ont été retenues parce que ce sont les seuls peuplements où tous les procédés de récolte sont représentés en nombre suffisant. L'écart existant un an après la coupe entre le procédé utilisant l'abatteuse-porteuse (procédé 1) et les autres procédés pour ce qui est de la distribution de la régénération résineuse a été comblé au cours des années suivantes (figure 2). Ce résultat est en accord avec l'augmentation graduelle du CDR observée par Frisque et Vézina (1977) au cours des 10 premières années suivant la coupe de peuplements résineux. Ainsi, il semble qu'un recrutement de semis résineux se produise généralement après la coupe dans les pessières à épinette noire pour augmenter le CDR des résineux de 15 à $25 \%$ (figure 2). Puisque le CDR est augmenté de 5 à $10 \%$ de plus en tenant compte des espèces feuillues commerciales (figure 2), il semble donc que la remise en production du groupement d'espèces EE ne pose généralement pas de problème même si le CDR immédiatement après la coupe est légèrement en deçà de $60 \%$.

Il est intéressant de noter la rapide progression du CDR des espèces feuillues commerciales et de leur position de dominance dans les placettes de régénération au cours des cinq premières années suivant la coupe (figure 2). Cette régénération en espèces feuillues provient de semenciers laissés sur pied lors de la récolte des peuplements. La plus haute taille que confere à la régénération résineuse son établissement avant la coupe semble donc constituer un avantage partiellement atténué par la croissance rapide du bouleau à papier et du peuplier faux-tremble qui sont les espèces feuillues généralement présentes dans le groupement d'espèces EE. À ce propos, Brumelis et Carleton
(1988) sont d'avis que la mécanisation de la récolte forestière a pour effet d'augmenter la composante feuillue des peuplements d'épinette noire parce que le passage de la machinerie modifierait suffisamment la surface du sol pour en diminuer le rapport $\mathrm{C} / \mathrm{N}$, ce qui favoriserait la croissance des espèces pionnières. La progression de la composante feuillue semble toutefois s'être arrêtée à partir de la cinquième année après la coupe et la proportion de tiges résineuses dominantes tend même à s'accroître légèrement au détriment des feuillus 10 ans après la coupe (figure 2). Cette proportion de résineux dominants dans la strate de régénération pourrait être augmentée en appliquant des mesures visant à protéger la régénération prétablie de haute taille.

Par ailleurs, McInnis et Roberts (1994; 1995) ont fait état de la possibilité d'une amélioration du taux de survie des petits semis prétablis dans le cas d'un système d'exploitation intégrant l'ébranchage sur le site de récolte. En effet, leurs résultats suggèrent que les procédés de récolte ne laissant que peu de débris de coupe au sol pourraient augmenter la mortalité des petits semis puisque l'amplitude des écarts de température au sol serait augmentée à court terme et que la compétition exercée par des espèces pionnières s'intensifierait à plus long terme. Ce phénomène n'a cependant pas été observé dans le cadre de la présente étude puisque le CDR des résineux associé au procédé 4 (bois en longueur) a été semblable à celui des autres procédés (arbre entier) pour l'ensemble de la période d'échantillonnage (figure 2).

\section{3. Évolution de la régénération dans les autres groupements d'espèces}

La distribution et la composition de la régénération préétablie des peuplements résineux (figure 3 ) et mélangés (figure 4) montrent une nette domination du sapin baumier et de l'épinette noire, 


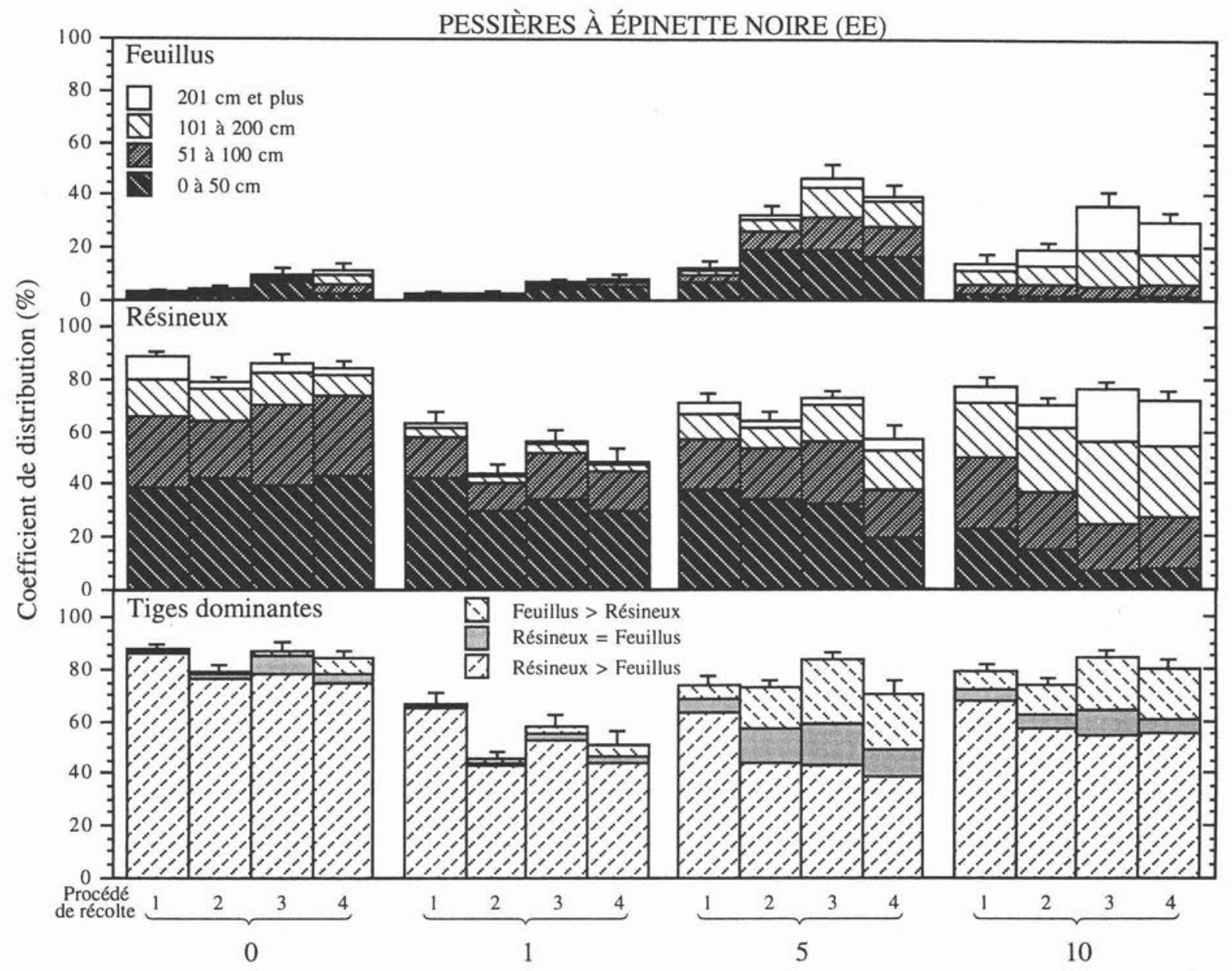

Nombre d'années après la coupe

Fig. 2. Coefficient de distribution de la régénération feuillue, résineuse et des tiges dominantes pour les quatre procédés de récolte utilisés en fonction du nombre d'années après la coupe.

deux espèces tolérantes à l'ombre. Si le CDR de l'épinette noire avant la coupe est généralement proportionnel à la surface terrière que cette espèce occupe dans le couvert dominant, ce n'est pas le cas du sapin baumier qui a tendance à augmenter sa proportion dans la strate de régénération par rapport au peuplement original (figures 3 et 4). La même tendance a déjà été notée par Hatcher (1960), Fye et Thomas (1963) et Vézina et Falardeau (1988). Cette réserve de semis de sapin sous le couvert dominant est d'ailleurs une caractéristique importante de la stratégie de régénération de cette espèce (Hatcher 1960; Morin 1994) qui ne semble pas pouvoir constituer de réserve de graines au sol à cause de leur courte période de viabilité (Houle et Payette 1991).

La présence d'une quantité appréciable de bouleau à papier dans la régénération prétablie de certains peuplements (figures 3 et 4 ) est plutôt inattendue puisque cette espèce est considérée comme étant intolérante à l'ombre. Une présence importante du bouleau à papier dans la strate de régénération préétablie est généralement associée aux peuplements ayant une composante importante en sapin baumier. Le bouleau à papier a la capacité de s'établir sous ce type de peuplement (Côté et Bélanger
1991), mais son développement ultérieur semble facilité par la création d'ouvertures dans le couvert causées par une défoliation du sapin par la tordeuse des bourgeons de l'épinette (Choristoneura fumiferana [Clem.]) (Ruel et Huot 1993).

L'examen des coefficients de distribution de la régénération préétablie révèle aussi que les stations dominées par le pin gris ( $\mathrm{PgPg}, \mathrm{PgE}$ et $\mathrm{PgPe}$ ) présentent des problèmes de remise en production parce que cette espèce ne s'établit pratiquement pas sous son propre couvert (figures 3 et 4). L'inventaire de régénération réalisé cinq ans après la coupe montre qu'une certaine quantité de semis de pin gris peut s'établir pendant cette période mais que leur coefficient de distribution, généralement sous les $20 \%$, n'est pas suffisant pour assurer la remise en production des superficies exploitées. Dans les peuplements les mieux régénérés, il serait donc important de prendre un soin particulier à protéger la régénération au moment de la récolte pour conserver la composante résineuse du futur peuplement et espérer atteindre une distribution adéquate de la régénération. C'est ce qui semble se réaliser dans le cas du groupement d'espèces $\mathrm{PgPe}$ puisque la protection de la régénération d'épinette noire a été suffisante pour que le CDR des tiges dominantes résineuses 


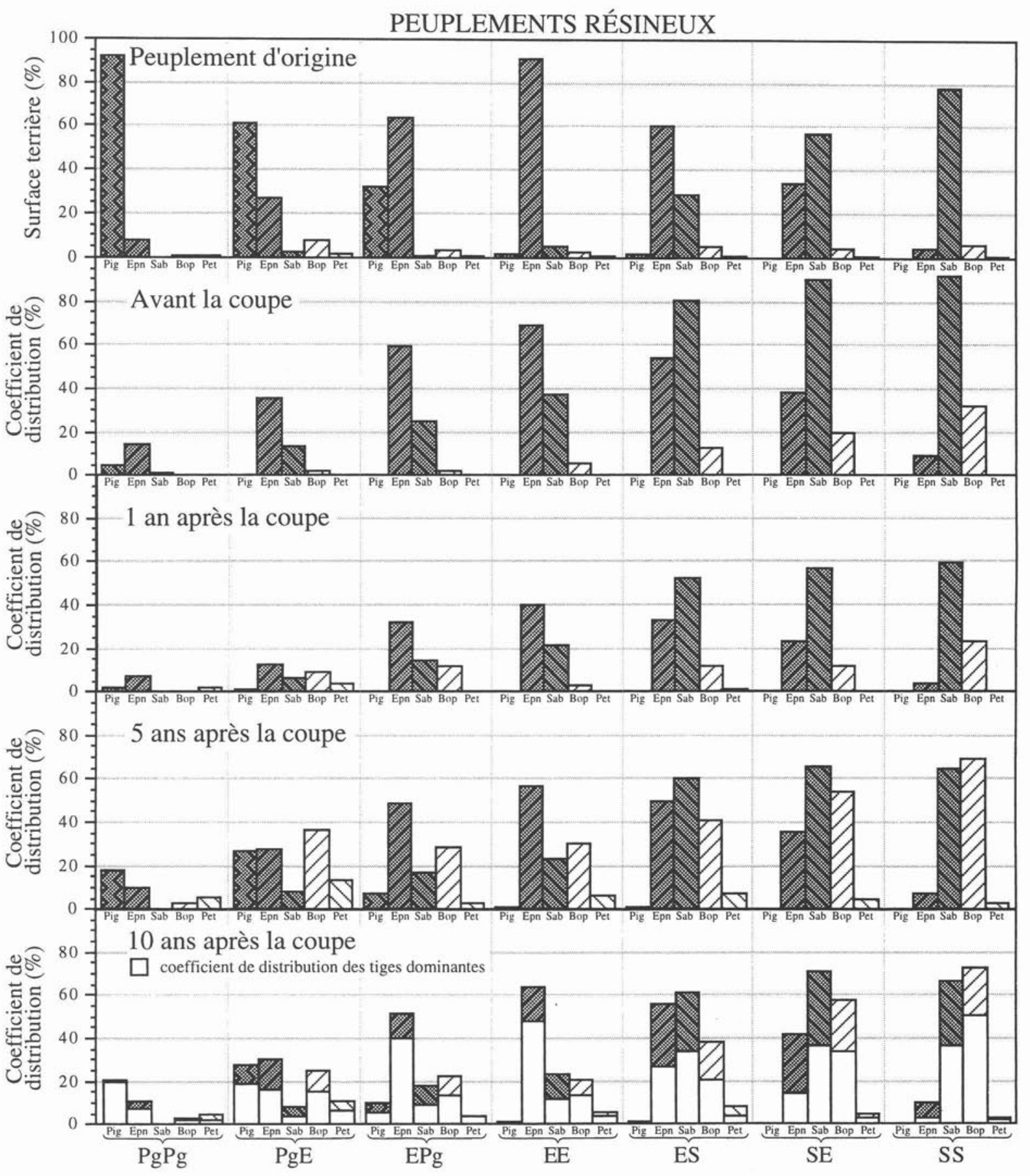

Groupement d'espèces

Fig. 3. Pourcentage de surface terrière du peuplement d'origine et coefficients de distribution de la régénération du pin gris (Pig), de l'épinette noire (Epn), du sapin baumier (Sab), du bouleau à papier (Bop) et du peuplier faux-tremble (Pet) en fonction du nombre d'années après la coupe pour les groupements résineux décrits au tableau 1 .

10 ans après la coupe soit semblable à la proportion qu'occupaient ces espèces dans le peuplement original (figure 4), ce qui a contribué à l'obtention d'un CDR de $73 \%, 10$ ans après la coupe (tableau 3). Cependant, la destruction marquée de la régénération d'épinette noire du groupement d'espèces $\mathrm{PgE} a$ fait en sorte que le $\mathrm{CDR}$ des résineux dominants 10 ans après la coupe n'est que de $37 \%$ (figure 3 ) alors que le CDR de toutes les espèces atteint $59 \%$ (tableau 3 ). Comparativement au peuplement d'origine, le groupement d'espèces PgE semble donc en voie d'augmenter sa proportion d'espèces feuillues au 


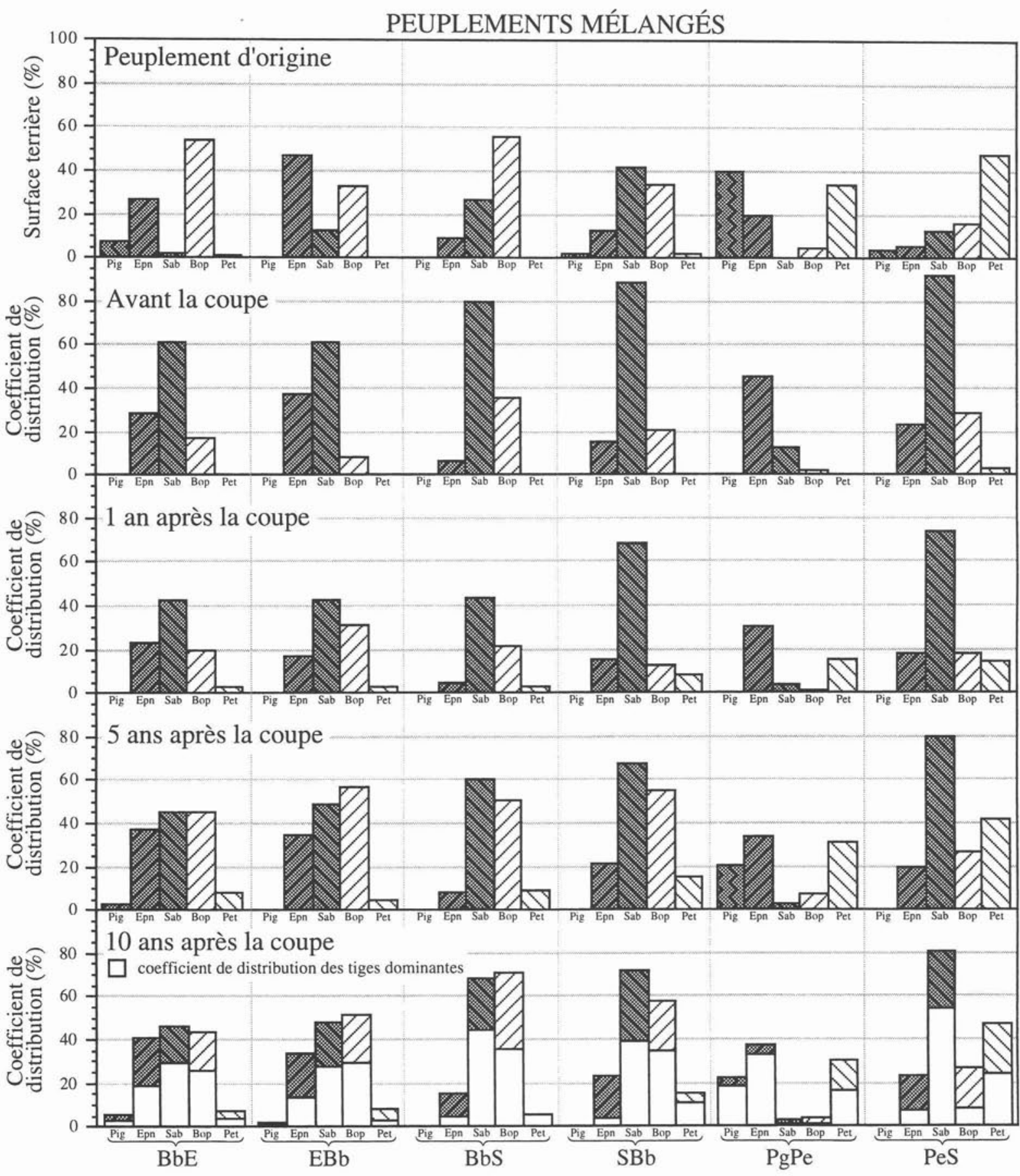

Groupement d'espèces

Fig. 4. Pourcentage de surface terrière du peuplement d'origine et coefficients de distribution de la régénération du pin gris (Pig), de l'épinette noire (Epn), du sapin baumier (Sab), du bouleau à papier (Bop) et du peuplier faux-tremble (Pet) en fonction du nombre d'années après la coupe pour les groupements mélangés décrits au tableau 1.

détriment des résineux.

Par ailleurs, les peuplements purs de pin gris (groupement d'espèces $\mathrm{PgPg}$ ) présentent d'évidentes lacunes de régénération puisque leur CDR n'atteignait que $30 \%, 10$ ans après la coupe (tableau 3). Même si ces peuplements sont généralement établis sur des stations pauvres, la croissance en hauteur du pin gris y est relativement élevée (tableau 3), ce qui suggère de favoriser l'établissement de cette espèce. Toutefois, puisqu'il n'y a que très peu de régénération préétablie sous ce type de couvert (figure 3 ), il semble que les modes de régénération à privilégier 
Tableau 3. Coefficient de distribution des espèces commerciales et non commerciales, nombre de tiges de plus de 5 cm de hauteur, hauteur et croissance en hauteur des tiges dominantes, 10 ans après la coupe

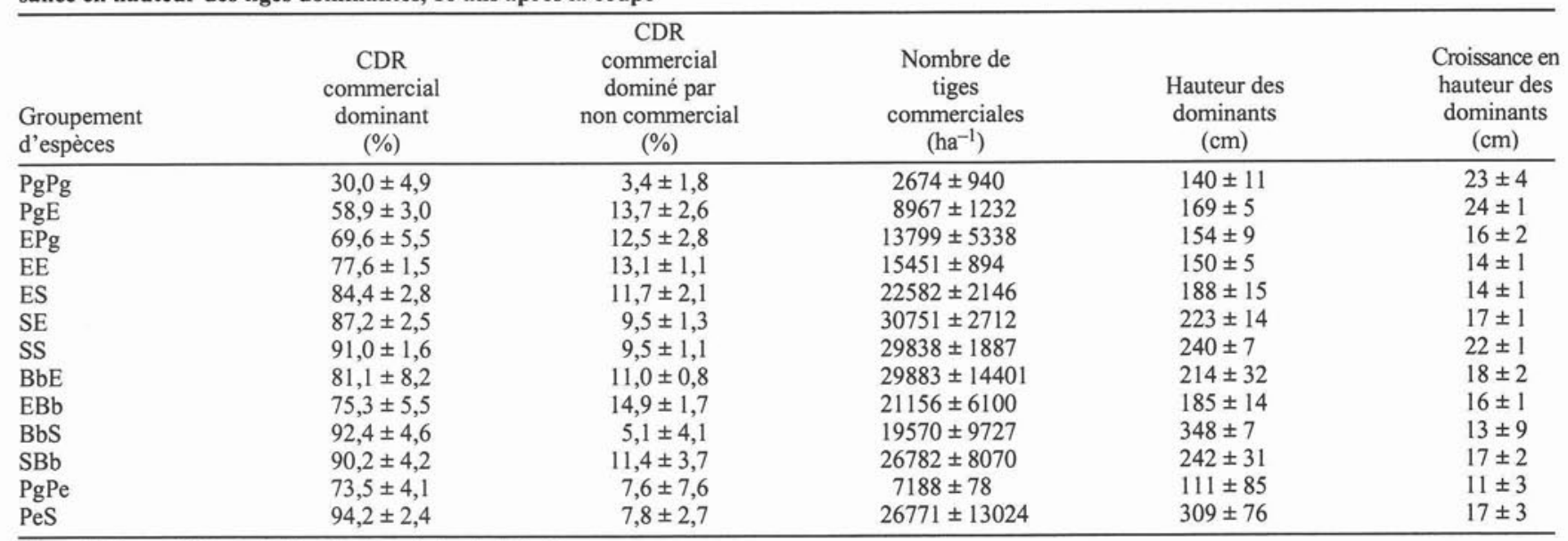

Note: CDR: coefficient de distribution de la régénération.

dans ce cas soient la plantation, le regarni ou l'ensemencement.

L'examen du CDR des tiges dominantes 10 ans après la coupe des peuplements résineux indique que la composante d'espèces feuillues a tendance à augmenter par rapport au peuplement original pour la plupart des groupements d'espèces (figure 3) comme l'ont déjà remarqué Frisque et al. (1978). Il semble même que les groupements d'espèces SE et SS évolueraient vers des peuplements mélangés si la composition des peuplements à maturité était fidèle à la composition des tiges dominantes retrouvées 10 ans après la coupe. Les observations de Vézina et Falardeau (1988) ne supportent toutefois pas cette hypothèse puisque l'évolution de peuplements correspondant aux groupements d'espèces ES et SE montre que le nombre de tiges d'espèces feuillues augmente rapidement pendant les 10 premières années qui ont suivi la coupe à blanc pour ensuite diminuer progressivement. Les raisons de ce déclin sont peut-être liées à une augmentation graduelle de la croissance en hauteur des espèces résineuses qui leur aurait permis de dominer la végétation concurrente, comme l'a remarqué Wall (1983). Quoiqu'il en soit, même si les feuillus gardaient leur position dominante 15 ans après la coupe, le CDR des résineux est suffisamment élevé dans ces groupements d'espèces (figure 3) pour permettre aux aménagistes d'ajuster la composition des peuplements en fonction de leurs besoins à l'aide d'une éclaircie précommerciale. Cette intervention semble d'ailleurs inévitable dans les peuplements à forte composante de sapin parce qu'ils comportent généralement plus de 20000 tiges/ha et qu'environ $10 \%$ des placettes régénérées par une espèce commerciale sont dominées par des espèces non commerciales (tableau 3).

Les résultats obtenus 10 ans après la coupe laissent entrevoir d'autres modifications dans la composition des peuplements régénérés comparativement au groupe d'espèces original. Ainsi, le groupement d'espèces ES, dont la surface terrière était composée de $60 \%$ d'épinette noire et de $28 \%$ de sapin, est maintenant dominé par le sapin (33\%) qui devance l'épinette noire (26\%) (figure 3). Cette tendance, qui semble confirmée par les résultats à long terme de Vézina et Falardeau (1988), ferait en sorte que le groupement d'espèces SE succéderait à des peuplements initialement identifiés comme ES si aucune intervention (e.g. éclaircie précommerciale) ou perturbation naturelle ne survenait jusqu'à la maturité de ces peuplements. Sur certaines stations, toutefois, la domination du sapin ne pourrait être que temporaire puisque Doucet et Boily (1995) ont remarqué un déclin de la croissance en hauteur du sapin cinq ans après la coupe alors que celle de l'épinette noire progressait continuellement pendant les 10 premières années suivant la coupe.

La composition des peuplements mélangés établis après la coupe pourrait aussi différer de celle du groupement d'espèces initial. En effet, les peuplements mélangés à prédominance feuillue $(\mathrm{BbE}, \mathrm{BbS}$ et $\mathrm{PeS})$ ont tendance à évoluer vers des peuplements mélangés à prédominance résineuse à cause de la composition de la régénération préétablie qui est dominée par des conifëres (figure 4). Pour les mêmes raisons, les peuplements mélangés à prédominance résineuse $(\mathrm{EBb}, \mathrm{SBb}$ et $\mathrm{PgPe})$ tendent à conserver le même type de composition après la coupe (figure 4). Il faut toutefois remarquer que les données tirées des peuplements mélangés doivent être utilisées avec précaution étant donné le petit nombre de peuplements échantillonnés (tableau 1).

\section{Conclusion}

La baisse du coefficient de distribution de la régénération de 25 à $40 \%$ observée dans cette étude est typique des systèmes d'exploitation et des méthodes de travail utilisés depuis le début de la mécanisation des opérations forestières. Malgré cette destruction importante de la régénération préétablie, une remise en production adéquate de la plupart des aires de coupe peut être constatée à cause du recrutement de semis qui s'est produit au cours des dix premières années suivant la coupe. Les peuplements ne comportant que peu de régénération préétablie ont toutefois présenté des niveaux de régénération déficients après la coupe, ce qui suggère d'utiliser des méthodes de protection de la régénération pendant les activités de récolte. Les porteurs forestiers seraient notamment plus efficaces que les débusqueuses à câble pour protéger la régénération mais une réduction de la superficie de l'aire de coupe occupée par les sentiers de débardage serait une mesure plus importante à adopter parce qu'elle permettrait de sauvegarder la régénération de haute taille. Cette mesure devrait d'ailleurs être appliquée dans tous les peuplements, même dotés d'une abondante régénération préétablie, parce qu'une strate de régénération composée d'individus de haute taille leur 
permettrait de se soustraire plus rapidement à la compétition herbacée et arbustive, d'augmenter la composante résineuse des futurs peuplements et de diminuer la période de temps nécessaire à l'atteinte d'un volume donné à maturité. La méthode de récolte introduite dans la Stratégie de protection des forêts du Gouvernement du Québec et qui vise à protéger la régénération et le sol semble donc une mesure appropriée mais dont l'efficacité devra cependant être validée au cours des prochaines années.

\section{Remerciements}

Des remerciements s'adressent à André Proulx, Gilles Rhéaume, Claude Deschênes et Bruno Canuel pour l'établissement de l'étude et la coordination des prises de mesures sur le terrain, à Mario Ménard pour son aide informatique et au personnel de plusieurs unités de gestion pour leur collaboration dans la prise de données. MM. René Doucet, Fabien Caron et Gilles Frisque ont aimablement révisé une version préliminaire du manuscrit et leurs commentaires ont été grandement appréciés.

\section{Bibliographie}

Arnott, J.T. 1968. Tree length — wheeled-skidder logging and its effects in certain black spruce forest types in Quebec. Pulp Pap. Mag. Can. 69: 103-109.

Brumelis, G. et T.J. Carleton. 1988. The vegetation of postlogged black spruce lowlands in central Canada. I. Trees and tall shrubs. Can. J. For. Res. 18: 1470-1478.

Côté, S. et L. Bélanger. 1991. Variations de la régénération préétablie dans les sapinières boréales en fonction de leurs caractéristiques écologiques. Can. J. For. Res. 21: 1779-1795

Doucet, R. et J. Boily. 1995. Croissance en hauteur de la régénération d'épinette noire et de sapin baumier après la coupe. Min. Res. Nat. Québec, Dir. Rech. For., Note Rech. For. 68.

Frisque, G. et P.E. Vézina. 1977. Reproduction de l'épinette noire (Picea mariana) après coupe à blanc de superficie réduite. Can. J. For. Res. 7: 648-655.

Frisque, G., G.F. Weetman et E. Clemmer. 1978. Analyse, 10 ans après coupe de bois à pâte, des problèmes de régénération dans l'Est du Canada. Institut canadien de recherches en génie forestier (FERIC). Rapport technique RT-23.

Froning, K. 1980. Logging hardwoods to reduce damage to white spruce understory. Envir. Can., Can. For. Serv., North. For. Res. Cent., Inf. Rep. NOR-X-229.

Fye, R.E. et J.B. Thomas. 1963. Regeneration of balsam fir and spruce about fifteen years following release by spruce budworm attack. For. Chron. 39: 385-397.

Gingras, J.-F. 1990. Les méthodes d'exploitation favorisant la protection de la régénération: l'expérience du Québec. Institut canadien de recherches en génie forestier (FERIC). Fiche Technique FT-144.
Gingras, J.-F., D. Cormier, J.-C. Ruel et D. Pin. 1991. Étude comparative de l'impact de trois modes de débardage sur la régénération préétablie. Institut canadien de recherches en génie forestier (FERIC). Fiche Technique FT 163.

Groot, A. 1995. Harvesting method affects survival of black spruce advance growth. North. J. Appl. For. 12: 8-11.

Hatcher, R.J. 1960. Development of balsam fir following a clearcut in Quebec. Dep. North. Aff. Nat. Res., For. Branch, For. Res. Div., Tech. Note 87.

Houle, G. et S. Payette. 1991. Seed dynamics of Abies balsamea and Acer saccharum in a deciduous forest of northeastern North America. Am. J. Bot. 78: 895-905.

Martin, C.W. 1988. Soil disturbance by logging in New EnglandReview and management recommendations. North. J. Appl. For. 5: 30-34.

McInnis, B.G. et M.R. Roberts. 1994. The effects of full-tree and tree-length harvests on natural regeneration. North. J. Appl. For. 11: 131-137.

McInnis, B.G. et M.R. Roberts. 1995. Seedling microenvironment in full-tree and tree-length logging slash. Can. J. For. Res. 25: 128-136. Ministère des Ressources naturelles du Québec. 1995. Norme de stratification écoforestière. Troisième programme de connaissance de la ressource forestière. Direction de la gestion des stocks forestiers, Québec. Morin, H. 1994. Dynamics of balsam fir forests in relation to spruce budworm outbreaks in the Boreal Zone of Quebec. Can. J. For. Res. 24: 730-741.

Pominville, P. 1993. Évolution quinquennale de la régénération protégée lors de récoltes par abattage manuel dans des sapinières. For. Chron. 69: 569-578.

Pothier, D., R. Doucet et J. Boily. 1995. The effect of advance regeneration height on future yield of black spruce stands. Can. J. For. Res. 25: 536-544.

Ruel, J.C. 1989. Importance de la régénération préexistante dans les forêts publiques du Québec. Ann. Sci. For. 46: 345-359.

Ruel, J.-C. 1992. Abondance de la régénération 5 ans après la coupe à blanc mécanisée de peuplements d'épinette noire (Picea mariana). Can. J. For. Res. 22: 1630-1638.

Ruel, J.-C. et M. Huot. 1993. Impact de la tordeuse des bourgeons de l'épinette [Choristoneura fumiferana (Clem.)] sur la régénération des sapinières après la coupe à blanc. For. Chron. 69: 163-172.

Vézina, P.-E. et H. Falardeau. 1988. Le développement de peuplements de coniferes sur une période de 50 ans après la coupe. For. Chron. 64: 329-333.

Wall, R.E. 1983. Early stand development after clear-cutting on the Cape Breton Highlands. Can. For. Serv., Maritimes For. Res. Cent., Inf. Rep. M-X-143.

Webber, B., J.T. Arnott, G.F. Weetman et G.C.R. Croome. 1969. Advance growth destruction, slash coverage and ground conditions in logging operations in eastern Canada. Pulp Pap. Res. Ins. Can., Wood. Pap. 8. 